\title{
On the physical interpretation of effective actions using Schwinger's formula
}

\author{
L. C. de Albuquerque*, C. Farina, Silvio J. Rabello and Arvind N. Vaidya \\ Instituto de Física, Universidade Federal do Rio de Janeiro, \\ Rio de Janeiro, RJ, Caixa Postal 68.528-CEP 21945-970, Brasil
}

(May 27, 2018)

\begin{abstract}
We show explicitly that Schwinger's formula for one-loop effective actions corresponds to the summation of energies associated with the zero-point oscillations of the fields. We begin with a formal proof, and after that we confirm it using a regularization prescription.
\end{abstract}

*e-mail: farina@vms1.nce.ufrj.br 
An explanation for the Casimir energy [1] is that it appears when one distorts the vacuum of a quantum field theory by introducing boundaries or background fields. One way to study this vacuum distortion is to perform the canonical quantization of the theory and the result is that this energy turns out to be the sum of zero-point oscillations of the quantum fields. In the functional approach the Casimir energy emerges as an effective potential induced by the vacuum fluctuations in the presence of these external perturbations [2].

The connection between these two points of view was explored in [3] and more recently studied in [4] with the aid of the $\zeta$-function method to evaluate the effective potential and turn it into a sum of zero-point energies.

In this paper we use the proper time formula derived long ago by Schwinger, in his early investigations on effective actions in QED [5], and recently applied by himself to the Casimir effect [6], to establish the connection of the effective potential with the zero-point oscillations. This formula is widely used in quantum field theory, in special when one is confronted with background fields as in the semiclassical approach to quantum gravity [7]. We first employ this formula (hereafter Schwinger's formula) in a rather formal way with regard to the divergences that appear during the calculations and then proceed in a regularized fashion, obtaining a regularized sum of zero-point energies.

Although the result we are going to prove is quite general, we choose for simplicity a definite problem in order to emphasize the basic points of our demonstration without dealing with involved mathematical details.

Consider a massive scalar field in a $(3+1)$-dimensional space-time (the extension to $d+1$ dimensions is trivial) constrained by two flat plates of area $\mathrm{A}$, orthogonal to the $\mathrm{z}$ axis and placed at $z=0$ and $z=a$ (we assume that $A \gg a^{2}$ ). The boundary conditions on the plates are chosen to be of the Dirichlet type so that the fields must vanish at $z=0$ and $z=a$.

In a recent paper [6] this energy was obtained, for the massless case, using the Schwinger's formula

$$
W_{0}=-\frac{i}{2} \operatorname{Tr} \int_{s_{0}}^{\infty} \frac{d s}{s} e^{-i s(H-i \epsilon)},
$$


where $W_{0}$ is the one-loop effective action and $H$ is the proper-time Hamiltonian for the system at hand. The symbol $\operatorname{Tr}$ refers to the trace over all the degrees of freedom, including those of spacetime.

Then, the Casimir energy is simply given by $\mathcal{E}=-\frac{W_{0}}{T}$, where $T$ can be viewed as a time interval. In equation (四), the regularizing cut-off $s_{0}$ must be put to zero only after a suitable subtraction of non-physical terms is made 円.

We first establish a formal connection and after that, we confirm our result in a more rigorous way, using a regularization procedure. Let us start with the formal expression with $s_{0}=0$ :

$$
W_{0}=-\frac{i}{2} \operatorname{Tr} \int_{0}^{\infty} \frac{d s}{s} e^{-i s(H-i \epsilon)}
$$

where $H=\mathbf{p}^{2}-\omega^{2}+m^{2}$, with $\mathbf{p}=-i \nabla, \omega=i \partial_{t}$. Taking a derivative with respect to $m^{2}$ on both sides of (2), performing the trace and making the identification $\mathcal{E}=-W_{0} / T$, we have

$$
\frac{1}{A} \frac{\partial \mathcal{E}}{\partial m^{2}}=\frac{1}{2} \sum_{n=1}^{\infty} \int \frac{d k_{1} d k_{2} d k_{0}}{(2 \pi)^{3}} \int_{0}^{\infty} d s e^{-i s\left(-k_{0}^{2}+k_{1}^{2}+k_{2}^{2}+\frac{n^{2} \pi^{2}}{a^{2}}-i \epsilon+m^{2}\right)} .
$$

Integration over $s$ immediately leads to

$$
\frac{1}{A} \frac{\partial \mathcal{E}}{\partial m^{2}}=\frac{i}{2} \sum_{n=1}^{\infty} \iint \frac{d k_{1} d k_{2}}{(2 \pi)^{2}} \int \frac{d k_{0}}{2 \pi} \frac{1}{k_{0}^{2}-\left[k_{1}^{2}+k_{2}^{2}+\frac{n^{2} \pi^{2}}{a^{2}}-i \epsilon+m^{2}\right]} .
$$

Now, with the aid of the residua theorem, we can integrate over $k_{0}$ to get

$$
\frac{1}{A} \frac{\partial \mathcal{E}}{\partial m^{2}}=\frac{1}{2} \sum_{n=1}^{\infty} \iint \frac{d k_{1} d k_{2}}{(2 \pi)^{2}} \frac{1}{\sqrt{k_{1}^{2}+k_{2}^{2}+\frac{n^{2} \pi^{2}}{a^{2}}+m^{2}}}
$$

Then, making the integration over $m^{2}$ we obtain,

$$
\frac{\mathcal{E}}{A}=\sum_{n=1}^{\infty} \iint \frac{d k_{1} d k_{2}}{(2 \pi)^{2}} \frac{1}{2} \omega\left(k_{1}, k_{2}, n\right)
$$

\footnotetext{
${ }^{1}$ An application of this formula for the same problem but with a different regularization prescription can be found in [8]. See also [9] for the massive case.
} 
apart from an additive irrelevant constant, and where we defined the eigenfrequencies (zeropoint energies of the field) $\omega\left(k_{1}, k_{2}, n\right)=\sqrt{k_{1}^{2}+k_{2}^{2}+\frac{n \pi}{a}^{2}+m^{2}}$. Expression (6) is precisely the usual (unregularized) mode summation expression for the Casimir energy of a massive scalar field between two parallel plates distant apart a distance $a$ [10].

Since in our previous deduction manipulations with divergent terms were made, let us now obtain the same result in a more rigorous way, what we mean is that we shall establish a connection between two regularized expressions, Schwinger's one and a (regularized) mode summation expression, with some kind of regularization prescription adopted.

However, instead of starting with the regularized expression (11) (with the cut-off $s_{0}$ ), we adopt the following regularization prescription

$$
W_{0}=-\frac{i}{2} \operatorname{Tr} \int_{0}^{\infty} d s s^{\nu-1} e^{-i s(H-i \epsilon)},
$$

where $\nu$ is large enough to make the integral well defined. In this approach, after the integral is computed an analytical continuation to the whole complex plane of $\nu$ must be done and then the limit $\nu \rightarrow 0$ must be carefully taken (sometimes an appropriate subtraction must be made, see [8,9] for more details on this approach)

As before we start by taking the derivative of (7) with respect to $m^{2}$. After evaluating the trace, we get

$$
\frac{\partial W_{0}}{\partial m^{2}}=-\frac{A T}{2} \sum_{n=1}^{\infty} \int \frac{d k_{1} d k_{2} d k_{0}}{(2 \pi)^{3}} \int_{0}^{\infty} d s s^{\nu} e^{-i s\left(-k_{0}^{2}+k_{1}^{2}+k_{2}^{2}+\frac{n^{2} \pi^{2}}{a^{2}}+m^{2}\right)} .
$$

Using the definition of the Euler Gamma function, the integration over $s$ readily yields

$$
\frac{\partial W_{0}}{\partial m^{2}}=-\left(i^{\nu+1}\right) \frac{A T}{2} \Gamma(\nu+1) \sum_{n=1}^{\infty} \iint \frac{d k_{1} d k_{2}}{(2 \pi)^{2}} \int \frac{d k_{0}}{2 \pi}\left[k_{0}^{2}-\left(k_{1}^{2}+k_{2}^{2}+\frac{n^{2} \pi^{2}}{a^{2}}+m^{2}\right)\right]^{-(\nu+1)}
$$

Using now that

$$
\int_{-\infty}^{\infty} d x\left(x^{2}+\alpha^{2}\right)^{-\sigma}=\left(\alpha^{2}\right)^{\frac{1}{2}-\sigma} B\left(\frac{1}{2}, \sigma-\frac{1}{2}\right)
$$

where $B(p, q)=\frac{\Gamma(p) \Gamma(q)}{\Gamma(p+q)}$, we get 


$$
\frac{\partial W_{0}}{\partial m^{2}}=-\left(i^{-\nu}\right) \frac{A T}{2} \Gamma\left(\frac{1}{2}\right) \Gamma\left(\nu+\frac{1}{2}\right) \sum_{n=1}^{\infty} \iint \frac{d k_{1} d k_{2}}{(2 \pi)^{2}} \frac{1}{2 \pi}\left[k_{1}^{2}+k_{2}^{2}+\frac{n^{2} \pi^{2}}{a^{2}}+m^{2}\right]^{-\left(\nu+\frac{1}{2}\right)} .
$$

Integrating on $m^{2}$ and identifying $\mathcal{E}=-\frac{W_{0}}{T}$, we finally obtain apart from an irrelevant additive constant

$$
\frac{\mathcal{E}}{A}=\sum_{n=1}^{\infty} \iint \frac{d k_{1} d k_{2}}{(2 \pi)^{2}} \frac{1}{2} \omega\left(k_{1}, k_{2}, n\right) F(\nu)
$$

where we defined

$$
F(\nu)=\frac{i^{-\nu}}{1-2 \nu} \frac{\Gamma\left(\nu+\frac{1}{2}\right)}{\sqrt{\pi}} \omega^{-2 \nu}\left(k_{1}, k_{2}, n\right)
$$

Some comments are in order here. Expression (12) is nothing but the usual mode summation with the presence of the regulator function $F(\nu)$, since $F(\nu)$ contains the negative power $\omega^{-2 \nu}$. So, for $\nu$ large enough expression (12) is well defined and after an analytical continuation to the whole complex $\nu$ plane is made, the limit $\nu \rightarrow 0$ can be (carefully) taken to yield the physical Casimir energy per unit area. It is easily seen that as $\nu \rightarrow 0$ we have that the regulator $F(\nu) \rightarrow 1$. Hence, we have established the equivalence between the modified Schwinger's formula (7) and the (regularized) mode summation approach. Although we have chosen a definite problem, i.e. the Casimir energy for a massive scalar field with Dirichlet boundary condition in one direction, our deduction is general, and therefore the Schwinger's formula for the (one-loop) effective action can always be interpreted as a summation over zero-point energies.

One of us (CF) would like to thank M. Asorey, A. J. Seguí-Santonja and M. V. Cougo Pinto for helpful discussions on this subject. This work was partially supported by CAPES and $\mathrm{CNPq}$ (Brazilian councils of research). 


\section{REFERENCES}

[1] H. G. B. Casimir, Proc. K. Ned. Akad. Wet 51, 793 (1948)

[2] W. Dittrich and M. Reuter, Effective Lagrangians in Quantum Electrodynamics, Lecture Notes In Physics, 220 (Springer, Berlin, 1985)

[3] C. Bernard, Phys. Rev. D 9, 3312 (1974)

[4] E. Myers, Phys. Rev. Lett. 59, 165 (1987)

[5] J. Schwinger, Phys. Rev. 82, 664 (1951)

[6] J. Schwinger, Lett. Math. Phys. 24, 59 (1992)

[7] N.D. Birrel and P.C.W. Davies, Quantum Fields in Curved Space (Cambridge University Press, Cambridge, 1982)

[8] M. V. Cougo-Pinto, C. Farina and A. J. Seguí-Santonja, Lett. Math. Phys. (1994), in press

[9] M. V. Cougo-Pinto, C. Farina and A. J. Seguí-Santonja, to appear in Lett. Math. Phys. (1994)

[10] Plunien, G., Muller, B. and Greiner, W., Phys. Rep. 134, 87 (1986) 